\section{BURNOUT SYNDROME AMONG PEDIATRICIANS: A CASE CONTROL STUDY COMPARING PEDIATRIC INTENSIVISTS AND GENERAL PEDIATRICIANS}

\author{
R.G. Branco ${ }^{1}$, T.T. Garcia², M.E. Molon², \\ P.C.R. Garcia ${ }^{3}$, J.P. Piva ${ }^{3}$, P.E. Ferreira ${ }^{4}$ \\ ${ }^{1}$ Paediatrics, Cambridge University, Cambridge, \\ UK, ${ }^{2}$ Department of Pediatrics, Pontificia \\ Universidad Católica do Rio Grande do Sul, \\ ${ }^{3}$ Paediatric Intensive Care Unit, Hospital Sao \\ Lucas da PUCRS, ${ }^{4}$ Department of Psychiatry, \\ Pontificia Universidad Católica do Rio Grande do \\ Sul, Porto Alegre, Brazil
}

Background and aims: Burnout Syndrome is a response to chronic occupational stress that may affect to many professional categories. It leads to low quality professional care, poor doctorpatient relationship, decreased ability to express empathy and increased medical errors. We aimed to estimate the prevalence of Burnout Syndrome among pediatric intensivistsand compare to a group of general pediatricians.

Methods: a case control study was conducted, selecting the pediatric intensivists working on duty in two regional and referral Brazilian PICU(PICU group) whereas an equal number of general pediatricians working with outpatients in the same hospitals were included as the control group(GPA group). Two blind researchers evaluated the both groups using the Portuguese version of the MBIMaslach Burnout Inventory scale. The protocol was approved by the PUCRS Ethics Committee.

Results: 35 cases and 35 controls were evaluated. No demographic differences between the groups were observed. Burnout was present in $71 \%$ of the PICU group and in $29 \%$ of the GPA group( $p<$ $0.01)$. The average score for all dimensions were higher for the emotional exhaustion(EE) and depersonalization(DE), and lower for professional accomplishment(PA) in the PICU group ( $P=0.0003)$. The multivariate analysis demonstrated that working in a PICU has independently associated with Burnout with an odds ratio 5.7(CI95\%;1.9-16.7) when compared to general pediatrician.

Conclusions: Burnout among pediatric intensivists is frequent and characteristically manifested by high levels of EE and DE and low levels of PA suggesting low quality of life, emotional suffering and professional dissatisfaction.

\section{PROLONGED RUPTURE OF MEMBRANES IN TERM INFANTS: SHOULD ALL BABIES BE SCREENED?}

\author{
C. Flannigan', M. Hogan²
}

${ }^{1}$ Neonatology, Royal Jubilee Maternity Hospital, Belfast, 'Neonatology, Craigavon Area Hospital, Portadown, UK

Background: Prolonged rupture of membranes is a major risk factor for early onset sepsis in neonates. In Northern Ireland there is no consistency on the management of this problem and individual clinical guidelines vary widely between neonatal departments. A present in Craigavon Hospital all term babies born with prolonged rupture of membranes have screening bloods performed.

Setting: The neonatal department of Craigavon Hospital a district general hospital in Northern Ireland.

Objectives: To determine if the current guidelines are being followed and to try and determine if the decision on whether to perform screening bloods was left up to the individual doctor's clinical judgement, would they make a safe decision.

Design: A prospective audit was carried out between October 2008 and January 2009.

Results: At present there is $100 \%$ compliance with the current guidelines and there is evidence that if the decision of whether to perform screening bloods is left up to the individual doctor's clinical judgement, they will make a sensible decision based on the infants risk factor assessment. None of the infants that the doctor decided they wouldn't screen, if it were not the policy to have done so, would have come to any harm if they hadn't had screening bloods performed.

Conclusion: Combining the results of the audit and the availability of nationally recognised guidelines it was decided to adopt the NICE guidelines in Craigavon Hospital. To help facilitate this change a Neonatal Early Warning Score observation chart has been developed to record the observations recommended by NICE. 